\title{
Why the Study of Cancer Needs a Systemic Approach?
}

\section{Fabio Grizzi*}

Laboratory of Molecular Gastroenterology, Humanitas Clinical and Research Center, Milan, Italy

Cancer is one of the most complex diseases affecting human beings and, despite the advances that have been made in molecular and cell biology, it is still unclear how cancer cells progress through carcinogenesis to acquire metastatic ability [1]. It is known that cancer is a clonal disease caused by the uncontrolled growth of a single genetically damaged cell, and that the malignant daughter cells within a clone interact with each other and their normal neighbouring cells in a microenvironment that influences tumour progression and invasion [2]. Furthermore, it is clear that cancer operates at different levels of the human hierarchical organisation, and evolves through a series of states (or possible pattern configurations) and transitions between states [3].

Most molecular biology-based studies reflect the reductionist view that genomic changes are all that is necessary to transform a normal cell into a neoplastic cell, a "conviction" that is based on the fact that the genome carries the information relating to all cell processes [4,5]. However, although this approach provides a still fundamental means of generating knowledge, there are a number of intriguing questions that remain to be answered. How many distinct regulatory pathways within each type of target cell need to be disrupted before the cell becomes cancerous? Is the same set of cell regulatory pathways disrupted in all of the neoplasias affecting humans? If we knew all of the molecular and cellular reactions occurring within a living normal cell and its tumoral counterpart, would we understand that cell?

Cartesian reductionism attempts to explain the variety of natural phenomena on the basis of the behaviour of a limited number of elements that are subject to simple but rigorous laws, and the progress that has been made in molecular and cell biology clearly shows that it has been a powerful driving force in science. However, there is still much to be done before what we know can be transformed into an understanding of the highly complex nature of genes, sub-cellular organelles, cells, tissues, organs, systems, and the body as a complete hierarchical system. The completion of the various genome projects has underlined the need to be able to approach systemic complexity more effectively, including the complex processes underlying the onset, progression and metastasis of cancer.

Human cancer is highly heterogeneous: there are more than 100 distinct types of human cancer, and various subtypes can be found inside specific organs. This genetic and phenotypical variability is the main cause of the self-progression and treatment response of a neoplastic disease [6], although the asynchrony [7] and self-progression of a cancer cell population suggests that the extent to which each neoplastic cell shares the properties of a normal cell differ in time and space [3]. The individual cells in a clonal population may respond differently (or not all) to the same stimulus, and this variability can also have important implications: [8] for example, although chemotherapy often reduces tumour size at first, it can give rise to severe adverse effects that lead to other complications [9]. It has also been shown that patients in a heterogeneous population can have a multiplicity of genetic variations that respond differently to a given medical intervention, and that the same treatment may benefit some but be harmful to others.

Carcinogenesis is a process affecting anatomical structures that have a virtually infinite number of parts and are governed by a large number of temporo-spatially different biological sub-processes (i.e. multi-scale causality). This means that: a) the relationships between the parts are not linear; b) the variables influencing the behaviour of the process are interrelated in a complex manner; c) the individual components are systematically heterogeneous; and d) small alterations in the variables can lead to completely different outcomes. All of these characteristics are reflected by differences in the progression or therapeutic response of human cancers, and the fact that histopathology does not always reveal their underlying biological behaviours. Every cancer treatment can be seen as a filter that removes the sub-population of sensitive cancer cells while allowing the insensitive sub-populations to escape.

In order to understand human cancer as a complex system involving many interacting components, it is necessary to establish the type of data that needs to be collected at each level of organisation, the boundary conditions for describing the disease (i.e. a perturbed system), and the technologies and approaches that are most capable of identifying its underlying biological behaviour [10]. We need to reanalyse traditional clinical concepts critically, reinterpret the clinical significance of failed therapies in terms of complexity, and consider multi-scale causality $[11,12]$ and cancer heterogeneity [13-16] when generating new medical interventions. It is likely that more can be discovered about the underlying behavioural characteristics of cancer if it is seen as a system that is dynamically complex in time and space, and reaching a quantitative understanding of this complexity will require the joint contributions of mathematicians, biologists and clinicians. Such a multidisciplinary approach can help to clarify old concepts, categorise our current knowledge, and suggest alternative methods of discovering new and clinically useful biomarkers.

\section{References}

1. Hanahan D, Weinberg RA (2011) Hallmarks of cancer: the next generation Cell 144: 646-674.

2. Marsh T, Pietras K, McAllister SS (2012) Fibroblasts as architects of cancer pathogenesis. Biochim Biophys Acta S09025-S4439, 00250-00255.

3. Grizzi F, Chiriva-Internati M (2006) Cancer: looking for simplicity and finding complexity. Cancer Cell Int 6:4.

4. Nurse P (1997) Reductionism: The ends of understanding. Nature 387: 657.

5. Szathmary E, Jordan F, Pal C (2001) Molecular biology and evolution. Can genes explain biological complexity? Science 292: 1315-1316.

6. Yates LR, Campbell PJ (2012) Evolution of the cancer genome. Nat Rev Genet 13: $795-806$.

7. Khan IA, Lupi M, Campbell L, Chappell SC, Brown MR, et al. (2011) Interoperability of time series cytometric data: a cross platform approach for modeling tumor heterogeneity. Cytometry A 79: 214-226.

8. Bastiaens $P$ (2009) Systems biology: When it is time to die. Nature 459: 334 335

*Corresponding author: Fabio Grizzi, Laboratory of Molecular Gastroenterology, Humanitas Clinical and Research Center, Via Manzoni 56, 20089 Rozzano, Milan Italy, E-mail: fabio.grizzi@humanitasresearch.it

Received November 26, 2012; Accepted November 27 2012; Published November 29, 2012

Citation: Grizzi F (2012) Why the Study of Cancer Needs a Systemic Approach? Pharmaceut Reg Affairs 1:e119. doi:10.4172/2167-7689.1000e119

Copyright: (c) 2012 Grizzi F. This is an open-access article distributed under the terms of the Creative Commons Attribution License, which permits unrestricted use, distribution, and reproduction in any medium, provided the original author and source are credited. 
9. Heng $\mathrm{HH}$ (2008) The conflict between complex systems and reductionism JAMA 300: 1580-1581.

10. Winslow RL, Trayanova N, Geman D, Miller MI (2012) Computational medicine: translating models to clinical care. Sci Transl Med 4: 158rv111.

11. Tortolina L, Castagnino N, De Ambrosi C, Moran E, Patrone F, et al. (2012) A multi-scale approach to colorectal cancer: from a biochemical- interaction signaling-network level, to multi-cellular dynamics of malignant transformation. Interplay with mutations and onco-protein inhibitor drugs. Curr Cancer Drug Targets 12: 339-355.

12. Enderling H, Chaplain MA, Hahnfeldt $P$ (2010) Quantitative modeling of tumor dynamics and radiotherapy. Acta Biotheor 58: 341-353.
13. Almendro V, Marusyk A, Polyak K (2012) Cellular Heterogeneity and Molecular Evolution in Cancer. Annu Rev Pathol.

14. Samuel N, Hudson TJ (2012) Translating Genomics to the Clinic: Implications of Cancer Heterogeneity. Clin Chem.

15. Horswell S, Matthews N, Swanton C (2012) Cancer heterogeneity and "The Struggle for Existence": Diagnostic and analytical challenges. Cancer Let S0304-S38358, 00636-00642.

16. Swanton C (2012) Intratumor Heterogeneity: Evolution through Space and Time. Cancer Res 72: 4875-4882. 